\title{
umacure
}

\section{Thoracic radiotherapy improves survival in small-cell lung cancer}

Patients with small-cell lung cancer typically present with extensive-stage disease (ESSCLC), which has a poor prognosis that has improved little over the past decades. Now, a trial by Ben Slotman et al. indicates that adding thoracic radiotherapy (TRT) usually reserved for symptom palliation-to standard therapy can improve survival.

In a previous trial of prophylactic cranial irradiation (PCI), Slotman et al. established that local radiotherapy can improve survival in ES-SCLC, while noting that intrathoracic tumours remain after therapy in $75 \%$ of the patients. "The next logical step was to see if TRT in these patients would lead to improved survival and improved intrathoracic control, without too much toxicity," says Slotman.

In the current phase III trial, patients with ES-SCLC that had responded to chemotherapy were randomized to receive either PCI only or PCI plus a 'high-palliative dose' of TRT (30 Gy in 10 fractions).
The addition of TRT significantly improved the 2-year overall survival (13\% versus 3\%; $P=0.004)$, although overall survival at 1 year was comparable $(33 \%$ versus $28 \%$ ). Furthermore, "improved progression-free survival [24\% versus $7 \%$ at 6 months], an almost $50 \%$ reduction in intrathoracic recurrences, and no serious toxicities were seen," states Slotman.

"We think that TRT should now be offered in addition to PCI to all patients with ES-SCLC who have a response after chemotherapy," Slotman concludes. He also identifies higher TRT doses, to further improve local control, and radiotherapy at other anatomical sites (such as the liver) as promising lines of future study.

\section{David Killock}

Original article Slotman, B. J. et al. Use of thoracic radiotherapy for extensive stage small-cell lung cancer: a phase 3 randomised controlled trial. Lancet doi:10.1016/S0140-6736(14)61085-0 